CASE REPORT

\author{
S. Muzzafar \\ L. Ketonen \\ J.S. Weinberg \\ D. Schellingerhout
}

\title{
Imaging and Clinical Features of an Intra-Axial Brain Stem Schwannoma
}

SUMMARY: A 68-year-old man presented with a highly symptomatic brain stem tumor originally thought to be a brain stem glioma. Intraoperative MR imaging guidance was used to resect the tumor, and real-time evoked potentials improved during surgery. Pathology findings unexpectedly indicated that the tumor was an intra-axial brain stem schwannoma, a condition reported, to our knowledge, only 6 times previously in the literature. The patient made an excellent recovery with reversal of his symptoms.

W

report an unusual case of a highly symptomatic intra-axial schwannoma that was successfully treated surgically.

\section{Case Report}

A 68-year-old man developed gait imbalance, coughing, and hiccups during a 2-month period. The symptoms progressed, and he developed nausea, vomiting, intermittent diplopia, and weakness in his right arm.

Physical examination revealed an alert well-oriented patient, with normally reacting pupils and intact extraocular movements. Positive findings included bilateral nystagmus on lateral gaze, right facial weakness, hoarse voice, and weakness in the right upper and lower extremity ( 4 of 5 weaknesses on the 5-point strength scale). Swallowing and motor speech assessment showed severe pharyngeal phase dysphagia with noneffective and unsafe swallowing.

MR imaging showed a cystic septate lesion involving the midbrain, pons, and medulla (Fig 1). The upper extent was in the left midbrain in the anterior pretectal region. The inferior extent was from the left medulla up to the anterior pyramids. The lesion was hypointense on T1 (not shown), hyperintense on T2 (Fig 2), and had enhancing septa on gadolinium-enhanced images (Fig 1). The cerebellar peduncles and anterior cerebellum on the left were also involved, showing fluid-attenuated inversion recovery signal-intensity abnormality (not shown).

The patient underwent a craniotomy with intraoperative MR imaging and sonography, with microscopic dissection guided by motorevoked potentials. The surface of the brain stem where the tumor came to the surface was abnormal, but there was no extension along any cranial nerve. A biopsy showed a pure spindle cell tumor. Pathologic considerations at the time were either meningioma, schwannoma, or tanycytic ependymoma. There were no mitotic figures. Xanthochromic intratumoral cyst fluid was found to be under moderate pressure. The tumor was resected but had to be coagulated medially and inferiorly at the brain stem attachment where dissection was difficult. Multiple septa were fenestrated and resected. Right-sided evoked potentials returned immediately once the cyst was decompressed. A gross total resection was achieved.

Pathologic results after the surgery showed a spindle cell neo-

Received May 12, 2009; accepted after revision May 22.

From the Neuroradiology Section (S.M., L.K., D.S.), Department of Radiology and Department of Neurosurgery (J.S.W.), University of Texas MD Anderson Cancer Center, Houston, Texas.

Please address correspondence to Dawid Schellingerhout, MD, Department of Radiology, MD Anderson Hospital, 1515 Holcombe Blvd, Houston, TX, 77030; e-mail: dawid. schellingerhout@di.mdacc.tmc.edu

DOI 10.3174/ajnr.A1758 plasm. Additional special studies were performed on permanent tissue. A positive tumor reactivity was seen in tumor cells with S-100, but they were negative for glial fibrillary acidic protein and epithelial membrane antigen. Studies for MIB-1 showed rare labeled tumor nuclei. A diagnosis of schwannoma was made.

The patient's neurologic status improved significantly almost immediately after surgery. His nystagmus resolved, swallowing function returned to normal, and the right-sided hemiparesis recovered. He is now neurologically healthy except for a trace left fourth-nerve weakness.

Postoperative MR imaging performed 11 months postsurgery showed persistent resolution of almost all the preoperative T2 signalintensity abnormality. A few small foci of extra-axial enhancement at the resection site were ascribed to postoperative changes. A small residual focus of T2 signal-intensity abnormality in the left cerebral peduncle and pons was seen.

\section{Discussion}

Intracranial schwannomas account for $8 \%$ of brain tumors ${ }^{1}$; most of these are acoustic neuromas, with intraparenchymal schwannomas being rare. Intraparenchymal schwannomas not arising from cranial nerves are uncommon and account for only $1 \%-2 \%$ of intracranial schwannomas. The most common sites are the frontal and temporal locations in the cerebral hemispheres. ${ }^{1}$ They have also been described in the cerebellar hemispheres and vermis ${ }^{2}$ in relation to the tentorium cerebelli and the lateral and fourth ventricles. ${ }^{3}$ More than 50 cases have been reported so far. ${ }^{4}$ Our review of literature revealed only 6 cases of intraparenchymal schwannomas located in the brain stem. ${ }^{1,5-9}$

Our case has unique and interesting features compared with other cases in the literature: 1) Our patient, at 68 years of age, tied with 1 other patient as the oldest on record (and this other elderly patient had an incidentally discovered lesion in the setting of ischemia ${ }^{9}$ ). 2) The high level of symptoms in our patient developed during 2 weeks; in the other 6 cases, symptoms developed from 6 weeks to 3 years. 3) Perhaps related to this acute presentation, our case is the second one ${ }^{10}$ with recorded T2 signal-intensity abnormality surrounding the lesion (of 3 cases with MR imaging available ${ }^{1,6,9}$ ). 4) Our patient had a multiloculated cystic lesion; all 3 other lesions ${ }^{1,6,9}$ with MR imaging data available had unilocular cystic masses. 5) Our case was 1 of only 2 lesions ${ }^{1}$ correctly diagnosed at the time of surgery by means of frozen sections. The other 5 cases $^{5-9}$ required permanent sections; thus, the diagnosis was made postprocedurally. 6) Unique to our case, continuous motor-evoked potentials recovered intraoperatively in our 


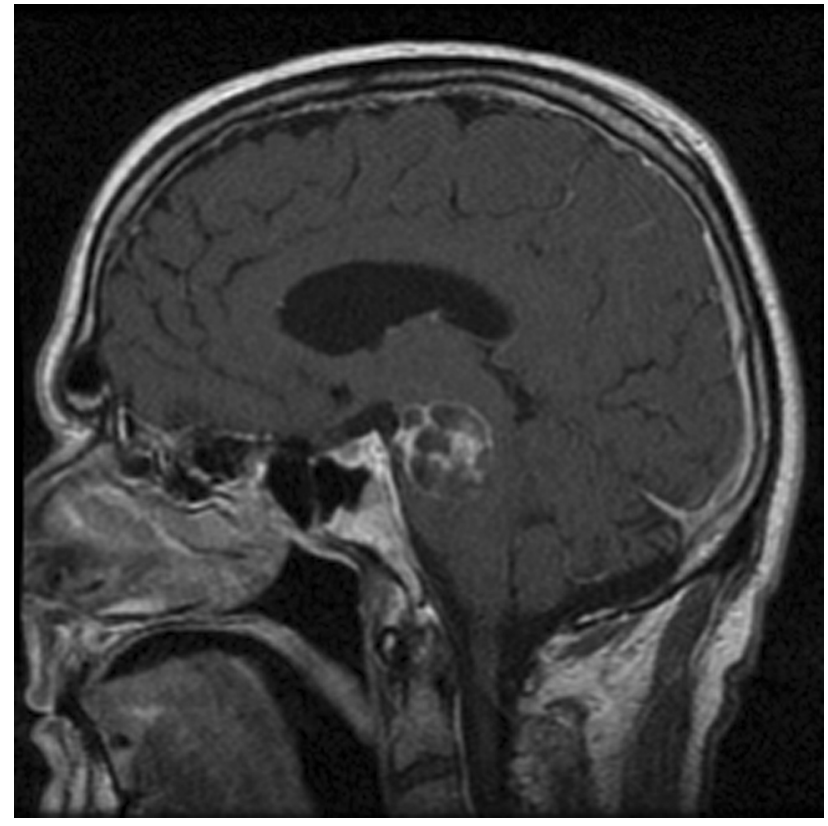

Fig 1. Sagittal T1-weighted enhanced image shows a well-demarcated cystic tumor with enhancing septa involving the midbrain and pons.

case. 7) Also, our patient was the first undergoing surgery in an integrated MR imaging operating suite environment, and 8) the tumor in our case was adherent to the brain stem, whereas in 3 cases, ${ }^{1,6,9}$ there was a well-developed capsule making the lesions easier to resect.

Our preoperative diagnosis was malignant glioma, a disease with a very poor prognosis, whereas the revised diagnosis of intraparenchymal schwannoma will likely have a considerably better outcome.

Schwannomas are well-circumscribed encapsulated masses usually found in relation to a nerve. Tumors form firm gray masses, and $15 \%-20 \%$ are associated with perilesional or peritumoral cysts. ${ }^{3}$ The tumors have well-demarcated irregular pushing rather than infiltrative margins. ${ }^{2}$

It is difficult to explain the origin of intraparenchymal brain schwannomas. Schwann cells are not normally present in the cerebral parenchyma. They have been detected around arteries in the intracranial perivascular nerve plexuses in the subarachnoid space and the brain. Similar tiny myelinated nerves may be present in the tela choroidea. ${ }^{2}$ Feigin and Ogata $^{11}$ have reported the presence of non-neoplastic Schwann cells in brain tissue in multiple sclerosis plaques (peripheral type myelin) or at the edge of old infarcts. These may undergo neoplastic conversion. Another hypothesis is that pial cells may sometimes undergo conversion to Schwann cells, which can be an origin of some intracerebral schwannomas. ${ }^{3}$

Characteristic imaging features show a well-circumscribed mass typically with cyst formation, a solid enhancing component, calcification, peritumoral edema, gliosis, and superficial or periventricular location. ${ }^{12}$ CT shows an isoattenuated or low-attenuation contrast-enhancing mass associated with a cyst. $^{2}$ The tumors are hypointense and hyperintense on T1and T2-weighted images, respectively. The solid component

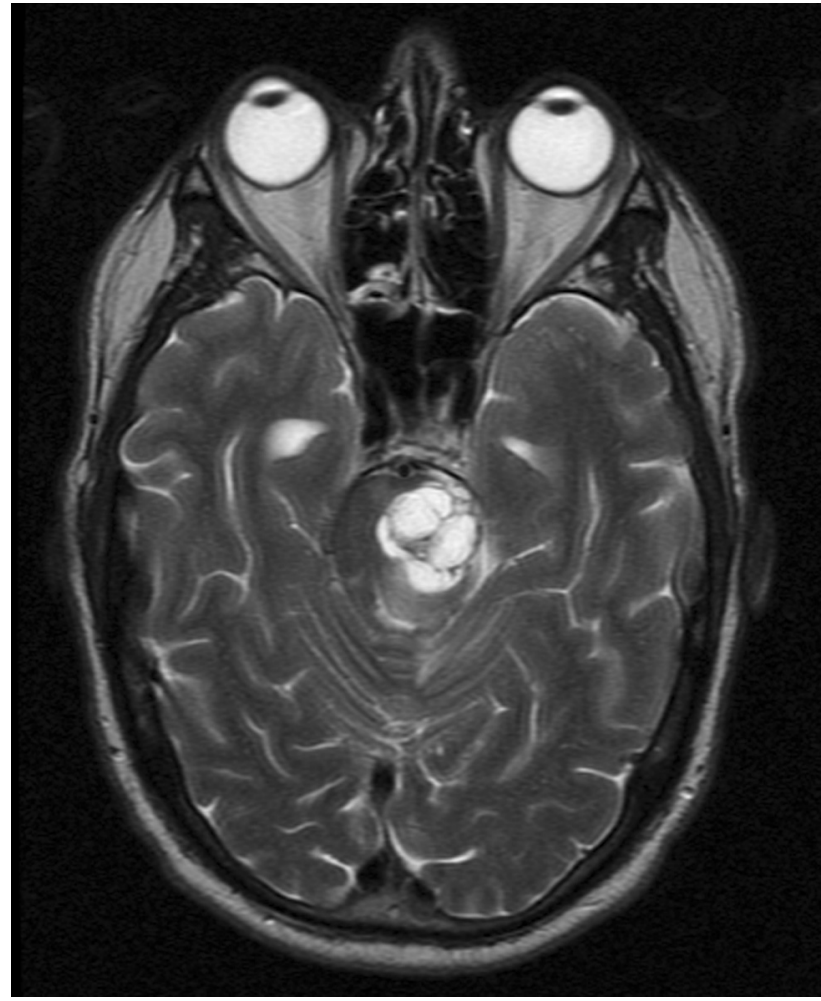

Fig 2. Axial T2-weighted image at the same level as in Fig 1 shows hyperintense signal intensity confirming the cystic nature of the tumor.

enhances strongly post-contrast administration. The cystic portion is a salient radiologic feature. ${ }^{2}$ It is thought that the pathogenesis of cyst formation is that small cysts within solid tumors derive from sites of microhemorrhage, necrosis, and degeneration, which fuse to become a larger cystic component. ${ }^{12}$ Such imaging features are also seen in schwannomas arising from cranial nerves.

The cystic degeneration rate of intracerebral schwannomas $(54.5 \%)$ is much higher than that of acoustic schwannomas $(9.6 \%-20.5 \%){ }^{4}$ Intramural nodules in the cyst wall have been reported rarely; absence of these nodules distinguishes them from cystic astrocytomas. ${ }^{7}$ The presence of calcification ${ }^{11}$ and frankly hemorrhagic lesions ${ }^{2}$ has also been reported. Calcification, seen on CT, may contribute to the hypoattenuated signal intensity on MR imaging. Diffuse peritumoral edema has been described as another characterstic feature of intraparenchymal schwannomas, especially in the supratentorial compartment $(35.7 \%))^{2,10,12}$ The cause of edema around this benign neoplasm is uncertain.

The outcome of patients with intraparenchymal schwannomas is typically very good. In their series of 9 cases, Casadei et $\mathrm{al}^{2}$ reported no evidence of tumor recurrence after complete resection in a follow-up period of 2-24 months with similar histologies, though there were no brain stem lesions in their series and some patients with subtotal resection did become symptomatic again.

Intra-axial schwannoma is difficult to distinguish from the main differential diagnosis of glioma a priori, particularly in the absence of cranial nerve involvement. The prominent neurologic symptoms may have been a clue, but the diagnosis in 
our case required open biopsy. Intraoperative frozen sections allowed the benign histology to be identified in time for the surgical approach to be modified, and a good functional outcome was obtained for the patient.

\section{References}

1. Lin J, Feng H, Li F, et al. Intraparenchymal schwannoma of the medulla oblongata: case report. J Neurosurg 2003;98:621-24

2. Casadei GP, Komori T, Scheithauer BW, et al. Intracranial parenchymal schwannoma: a clinicopathological and neuroimaging study of nine cases. J Neurosurg 1993;79:217-22

3. Osborn AB, Salzman K. Diagnostic Imaging: Brain. Salt Lake City: Amirsys; 2007

4. Luo B, Sun G, Zhang B, et al. Neuroradiological findings of intracranial schwannomas not arising from the stems of cranial nerves. Br J Radiol 2004;77: $1016-21$
5. Prakash B, Roy S, Tandon PN. Schwannoma of the brain stem: case report. J Neurosurg 1980;53:121-23

6. Aryanpur J, Long DM. Schwannoma of the medulla oblongata: case report. J Neurosurg 1988;69:446-49

7. Ladouceur D, Bergeron D, Lamarche JB, et al. Cystic schwannoma of the brainstem. Can J Neurol Sci 1989;16:357-60

8. Sharma V, Newton G. Schwannoma of the medulla oblongata. Br J Neurosurg 1993;7:427-29

9. Tanabe M, Miyata $\mathrm{H}$, Okamoto $\mathrm{H}$, et al. Brainstem schwannoma: case report. Neurol Med Chir (Tokyo) 1996;36:880-83

10. Tsuiki $\mathrm{H}$, Kuratsu $\mathrm{J}$, Ishimaru $\mathrm{Y}$, et al. Intracranial intraparenchymal schwannoma: report of three cases. Acta Neurochir (Wien) 1997;139:756-60

11. Feigin I, Ogata J. Schwann cells and peripheral myelin within human central nervous tissues: the mesenchymal character of Schwann cells. J Neuropathol Exp Neurol 1971;30:603-12

12. Zagardo MT, Castellani RJ, Rees JH, et al. Radiologic and pathologic findings of intracerebral schwannoma. AJNR Am J Neuroradiol 1998;19:1290-93 